\title{
SOME APPROXIMATION PROPERTIES FOR MODIFIED BASKAKOV TYPE OPERATORS
}

\author{
VIJAY GUPTA
}

\begin{abstract}
We study some direct results for the recently introduced family of modified Baskakov type operators. In particular, we obtain local direct results on ordinary and simultaneous approximation and an estimation of error for linear combinations in terms of higher order modulus of continuity. We have applied the Steklov mean as a tool for the linear approximating method.
\end{abstract}

2000 Mathematics Subject Classification: 41A30, 41A36.

Key words and phrases: Lebesgue integrable functions, direct results, simultaneous approximation, Steklov mean, linear combinations.

\section{INTRODUCTION}

Gupta et al. [5] introduced the integral modification of Baskakov operators to approximate Lebesgue integrable functions on the interval $[0, \infty)$ as

$$
G_{n}(f, x)=\int_{0}^{\infty} W(n, x, t) f(t) d t, \quad x \in[0, \infty),
$$

where the kernel $W(n, x, t)$ in terms of Dirac-delta functions is defined by

$$
W(n, x, t)=\sum_{\nu=1}^{\infty} p_{n, \nu}(x) b_{n, \nu-1}(t)+(1+x)^{-n} \delta(t),
$$

and

$$
p_{n, \nu}(x)=\left(\begin{array}{c}
n+\nu-1 \\
\nu
\end{array}\right) \frac{x^{\nu}}{(1+x)^{n+\nu}}, \quad b_{n, \nu}(t)=\frac{t^{\nu}}{B(\nu+1, n)(1+t)^{n+\nu+1}} .
$$

It is easily verified that the operators $G_{n}$ are linear positive operators. Also, $G_{n}(1, x)=1$.

Let $C_{B}[0, \infty)$ be the space of all real-valued continuous bounded functions $f$ on $[0, \infty)$ endowed with the norm $\|f\|=\sup _{x \geq 0}|f(x)|$. We consider the following $K$-functional:

$$
K_{2}(f, \delta)=\inf \left\{\|f-g\|+\delta\left\|g^{\prime \prime}\right\|: g \in W_{\infty}^{2}\right\}, \quad \delta>0,
$$

where $W_{\infty}^{2}=\left\{g \in C_{B}[0, \infty): g^{\prime}, g^{\prime \prime} \in C_{B}[0, \infty)\right\}$. Positive constants that may have different values at different occurences are denoted by $c$. By $[1$, p. 177 , Theorem 2.4] there exists $c>0$ such that

$$
K_{2}(f, \delta) \leq c \omega_{2}(f, \sqrt{\delta})
$$


where $\delta>0$ and

$$
\omega_{2}(f, \sqrt{\delta})=\sup _{0<h \leq \sqrt{\delta}} \sup _{x \in[0, \infty)}|f(x+2 h)-2 f(x+h)+f(x)|,
$$

is the second modulus of smoothness of $f \in C_{B}[0, \infty)$. Furthermore, let

$$
\omega(f, \delta)=\sup _{0<h \leq \delta} \sup _{x \in[0, \infty)}|f(x+h)-f(x)|,
$$

be the usual modulus of continuity of $f \in C_{B}[0, \infty)$.

Similar types of operators have recently been studied by Srivastava and Gupta [9]. We estimate local direct results in terms of modulus of smoothness and modulus of continuity in ordinary and simultaneous approximation. In the last section an error estimate is also established for linear combinations of the operators $G_{n}$ for an unbounded function with some growth property.

\section{Auxiliary Results}

We need the following lemmas in the sequel.

Lemma 2.1. Let the function $\mu_{n, m}(x), x \in[0, \infty)$, be defined as

$$
\mu_{n, m}(x)=\sum_{\nu=1}^{\infty} p_{n, \nu}(x) \int_{0}^{\infty} b_{n, \nu-1}(t)(t-x)^{m} d t+(-x)^{m}(1+x)^{-n} .
$$

Then

$$
\begin{aligned}
& \mu_{n, 0}(x)=1, \quad \mu_{n, 1}(x)=\frac{x}{n-1}, \quad n>1, \\
& \mu_{n, 2}(x)=\frac{x(x+1)(2 n-1)+(1+3 x) x}{(n-1)(n-2)}, \quad n>2,
\end{aligned}
$$

and for $n>m+1$ there holds the recurrence relation

$$
\begin{aligned}
(n-m-1) \mu_{n, m+1}(x)= & x(1+x)\left[\mu_{n, m}^{(1)}(x)+2 m \mu_{n, m-1}(x)\right] \\
& +[m(1+2 x)+x] \mu_{n, m}(x),
\end{aligned}
$$

which implies that

$$
\mu_{n, m}(x)=O\left(n^{-[(m+1) / 2]}\right)
$$

for each $x \in[0, \infty)$.

Proof. The values of $\mu_{n, 0}, \mu_{n, 1}(x)$ easily follow from the definition. We prove the recurrence relation

$$
\begin{aligned}
x(1+x) \mu_{n, m}^{(1)}= & \sum_{\nu=1}^{\infty} x(1+x) p_{n, \nu}^{(1)}(x) \int_{0}^{\infty} b_{n, \nu-1}(t)(t-x)^{m} d t \\
& -m \sum_{\nu=1}^{\infty} x(1+x) p_{n, \nu}(x) \int_{0}^{\infty} b_{n, \nu-1}(t)(t-x)^{m-1} d t \\
& -\left\{n(-x)^{m}(1+x)^{-n-1}+m(-x)^{m-1}(1+x)^{-n}\right\} x(x+1) .
\end{aligned}
$$


Now using the identities $x(1+x) p_{n, \nu}^{(1)}(x)=(\nu-n x) p_{n, \nu}(x)$ and $t(1+t) b_{n, \nu}^{(1)}(t)=$ $[\nu-(n+1) t] b_{n, \nu}(t)$, we obtain

$$
\begin{aligned}
x(1+ & x)\left[\mu_{n, m}^{(1)}(x)+m \mu_{n, m-1}(x)\right] \\
= & \sum_{\nu=1}^{\infty}(\nu-n x) p_{n, \nu}(x) \int_{0}^{\infty} b_{n, \nu-1}(t)(t-x)^{m} d t+n(-x)^{m+1}(1+x)^{-n} \\
= & \sum_{\nu=1}^{\infty} p_{n, \nu}(x) \int_{0}^{\infty} t(1+t) b_{n, \nu-1}^{(1)}(t)(t-x)^{m} d t \\
& +(n+1) \sum_{\nu=1}^{\infty} p_{n, \nu}(x) \int_{0}^{\infty} b_{n, \nu-1}(t)(t-x)^{m+1} d t \\
& +(1+x) \sum_{\nu=1}^{\infty} p_{n, \nu}(x) \int_{0}^{\infty} b_{n, \nu-1}(t)(t-x)^{m} d t+n(-x)^{m+1}(1+x)^{-n} \\
= & \sum_{\nu=1}^{\infty} p_{n, \nu}(x) \int_{0}^{\infty}\left[(1+2 x)(t-x)+(t-x)^{2}+x(1+x)\right] b_{n, \nu-1}^{(1)}(t)(t-x)^{m} d t \\
& +(n+1) \sum_{\nu=1}^{\infty} p_{n, \nu}(x) \int_{0}^{\infty} b_{n, \nu-1}(t)(t-x)^{m+1} d t \\
& +(1+x) \sum_{\nu=1}^{\infty} p_{n, \nu}(x) \int_{0}^{\infty} b_{n, \nu-1}(t)(t-x)^{m} d t+n(-x)^{m+1}(1+x)^{-n} \\
= & -[m(1+2 x)+x] \mu_{n, m}(x)+(n-m-1) \mu_{n, m+1}(x)-m x(1+x) \mu_{n, m-1}(x) .
\end{aligned}
$$

This completes the proof of the recurrence relation. The values $\mu_{n, 2}(x), \mu_{n, m}(x)$ follow from the recurrence relation.

Lemma 2.2. Let $n>r \geq 1$ and $f^{(i)} \in C_{B}[0, \infty)$ for $i \in\{0,1,2, \ldots, r\}$, then

$$
G_{n}^{(r)}(f, x)=\frac{(n+r-1) !(n-r-1) !}{((n-1) !)^{2}} \sum_{\nu=0}^{\infty} p_{n+r, \nu}(x) \int_{0}^{\infty} b_{n-r, \nu+r-1}(t) f^{(r)}(t) d t
$$

Proof. The following relation follows by simple calculation:

$$
\begin{aligned}
p_{n, \nu}^{(1)}(x) & =n\left[p_{n+1, \nu-1}(x)-p_{n+1, \nu}(x)\right] \\
b_{n, \nu}^{(1)}(t) & =n\left[b_{n+1, \nu-1}(t)-b_{n+1, \nu}(t)\right]
\end{aligned}
$$

where $x, t \in[0, \infty)$. 
Furthermore, we prove our lemma by mathematical induction. Using the above identities (3) and (4), we have

$$
\begin{aligned}
G_{n}^{(1)}(f, x)= & \sum_{\nu=1}^{\infty} p_{n, \nu}^{(1)}(x) \int_{0}^{\infty} b_{n, \nu-1}(t) f(t) d t-n(1+x)^{-n-1} f(0) \\
= & \sum_{\nu=1}^{\infty} n\left[p_{n+1, \nu-1}(x)-p_{n+1, \nu}(x)\right] \int_{0}^{\infty} b_{n, \nu-1}(t) f(t) d t-n(1+x)^{-n-1} f(0) \\
= & n(1+x)^{-n-1} \int_{0}^{\infty} n(1+t)^{-n-1} f(t) d t \\
& +n \sum_{\nu=1}^{\infty} p_{n+1, \nu}(x) \int_{0}^{\infty}\left(\frac{-1}{n-1}\right) b_{n-1, \nu}^{(1)}(t) f(t) f t-n(1+x)^{-n-1} f(0) .
\end{aligned}
$$

Applying the integration by parts, we get

$$
\begin{aligned}
G_{n}^{(1)}(f, x)= & n(1+x)^{-n-1} f(0)+n(1+x)^{-n-1} \int_{0}^{\infty}(1+t)^{-n} f^{(1)}(t) d t \\
& +\sum_{\nu=1}^{\infty} \frac{n}{n-1} p_{n+1, \nu}(x) \int_{0}^{\infty} b_{n-1, \nu}(t) f^{(1)}(t) d t-n(1+x)^{-n-1} f(0) \\
= & \frac{n}{n-1} \sum_{\nu=0}^{\infty} p_{n+1, \nu}(x) \int_{0}^{\infty} b_{n-1, \nu}(t) f^{(1)}(t) d t
\end{aligned}
$$

thus the result is true for $r=1$. The result easily follows by the principle of mathematical induction.

Lemma 2.3 (see [8], [2, p. 128]). For $m \in N \cup\{0\}$, if the $m$-th order moment is defined as

$$
U_{n, m}(x)=\sum_{\nu=0}^{\infty} p_{n, \nu}(x)\left(\frac{\nu}{n}-x\right)^{m},
$$

then $U_{n, 0}(x)=1, U_{n, 1}(x)=0$ and

$$
n U_{n, m+1}(x)=x(1+x)\left[U_{n, m}^{(1)}(x)+m U_{n, m-1}(x)\right] .
$$

Consequently, $U_{n, m}(x)=O\left(n^{-[(m+1) / 2]}\right)$.

Lemma 2.4 (see [2], [7], [8]). There exist polynomials $\phi_{i, j, r}(x)$ independent of $n$ and $\nu$ such that

$$
\{x(1+x)\}^{r} \frac{d^{r}}{d x^{r}}\left[p_{n, \nu}(x)\right]=\sum_{\substack{2 i+j \leq r \\ i, j \leq 0}} n^{i}[\nu-n x]^{j} \phi_{i, j, r}(x) p_{n, \nu}(x) .
$$




\section{Local Approximation}

In this section we establish direct local approximation theorems for operators (1).

Theorem 3.1. Let $f \in C_{B}[0, \infty)$. Then there exists an absolute constant $c>0$ such that

$$
\left|G_{n}(f, x)-f(x)\right| \leq c \omega_{2}\left(f, \sqrt{\frac{(x(1+x)}{n-1}}\right)+\omega\left(f, \frac{x}{n-1}\right)
$$

for every $x \in[0, \infty)$ and $n=3,4, \ldots$.

Proof. We define a new operator $\widehat{G}_{n}: C_{B}[0, \infty) \rightarrow C_{B}[0, \infty)$ as follows:

$$
\widehat{G}_{n}(f, x)=G_{n}(f, x)-f(x)+f\left(\frac{n x}{n-1}\right) .
$$

Then by Lemma 2.1 we obtain $\widehat{G}_{n}(t-x, x)=0$. Now, let $x \in[0, \infty)$ and $g \in W_{\infty}^{2}$. From Taylor's formula

$$
g(t)=g(x)+g^{\prime}(x)(t-x)+\int_{x}^{t}(t-u) g^{\prime \prime}(u) d u, \quad t \in[0, \infty),
$$

we get

$$
\begin{aligned}
\widehat{G}_{n}(g, x) & -g(x)=\widehat{G}_{n}\left(\int_{x}^{t}(t-u) g^{\prime \prime}(u) d u, x\right) \\
& =G_{n}\left(\int_{x}^{t}(t-u) g^{\prime \prime}(u) d u, x\right)+\int_{x}^{n x /(n-1)}\left(\frac{n}{n-1} x-u\right) g^{\prime \prime}(u) d u .
\end{aligned}
$$

On the other hand,

$$
\left|\int_{x}^{t}(t-u) g^{\prime \prime}(u) d u\right| \leq(t-x)^{2}\left\|g^{\prime \prime}\right\|
$$

and

$$
\begin{aligned}
\left|\int_{x}^{n x /(n-1)}\left(\frac{n}{n-1} x-u\right) g^{\prime \prime}(u) d u\right| & \leq\left(\frac{n x}{n-1}-x\right)^{2}\left\|g^{\prime \prime}\right\| \\
& \leq \frac{x^{2}}{(n-1)^{2}}\left\|g^{\prime \prime}\right\| \leq \frac{x(1+x)}{(n-1)^{2}}\left\|g^{\prime \prime}\right\| .
\end{aligned}
$$

Thus by (7), (8), (9) and by the positivity of $G_{n}$, we have

$$
|\widehat{G}(g, x)-g(x)| \leq G_{n}\left((t-x)^{2}, x\right)\left\|g^{\prime \prime}\right\|+\frac{x(1+x)}{(n-1)^{2}}\left\|g^{\prime \prime}\right\| .
$$


Hence in view of Lemma 2.1, we have

$$
\begin{aligned}
\left|\widehat{G}_{n}(g, x)-g(x)\right| & \leq\left(\frac{x(1+x)(2 n-1)+(1+3 x) x}{(n-1)(n-2)}+\frac{x(1+x)}{(n-1)^{2}}\right)\left\|g^{\prime \prime}\right\| \\
& \leq\left(\frac{2 n-1}{n-2}+\frac{3}{n-2}+\frac{1}{n-1}\right) \frac{x(1+x)}{n-1}\left\|g^{\prime \prime}\right\| \\
& \leq \frac{9}{(n-1)} x(1+x)\left\|g^{\prime \prime}\right\| .
\end{aligned}
$$

Again applying Lemma 2.1,

$$
\left|G_{n}(f, x)\right| \leq \sum_{\nu=1}^{\infty} p_{n, \nu}(x) \int_{0}^{\infty} b_{n, \nu-1}(t)|f(t)| d t+(1+x)^{-n}|f(0)| \leq\|f\| .
$$

This means that $G_{n}$ is a contraction, i.e. $\left\|G_{n} f\right\| \leq\|f\|, f \in C_{B}[0, \infty)$. Thus by $(6)$

$$
\left\|\widehat{G}_{n} f\right\| \leq\left\|G_{n} f\right\|+2\|f\| \leq 3\|f\|, \quad f \in C_{B}[0, \infty) .
$$

Using (6), (10) and (11), we obtain

$$
\begin{aligned}
& \left|G_{n}(f, x)-f(x)\right| \leq\left|\widehat{G}_{n}(f, x)-f(x)\right|+\left|f(x)-f\left(\frac{n x}{n-1}\right)\right| \\
& \quad \leq\left|\widehat{G}_{n}(f-g, x)-(f-g)(x)\right|+\left|\widehat{G}_{n}(g, x)-g(x)\right|+\left|f(x)-f\left(\frac{n x}{n-1}\right)\right| \\
& \quad \leq 4\|f-g\|+\frac{9}{n-1} x(1+x)\left\|g^{\prime \prime}\right\|+\left|f(x)-f\left(\frac{n x}{n-1}\right)\right| \\
& \quad \leq 9\left\{\|f-g\|+\frac{x(1+x)}{n-1}\left\|g^{\prime \prime}\right\|\right\}+\sup _{t, t-(x /(n-1)) \in[0, \infty)}\left|f\left(t-\frac{x}{n-1}\right)-f(t)\right| \\
& \quad \leq 9\left\{\|f-g\|+\frac{x(1+x)}{n-1}\left\|g^{\prime \prime}\right\|\right\}+\omega\left(f, \frac{x}{n-1}\right) .
\end{aligned}
$$

Now taking the minimum on the right-hand side over all $g \in W_{\infty}^{2}$ and using (2) we obtain the required result.

Theorem 3.2. Let $n>r+2 \geq 3$ and $f^{(i)} \in C_{B}[0, \infty)$ for $i \in\{0,1, \ldots, r\}$. Then

$$
\begin{aligned}
& \left|G_{n}^{(r)}(f, x)-f^{(r)}(x)\right| \leq\left(\frac{(n+r-1) !(n-r-1) !}{((n-1) !)^{2}}-1\right)\left\|f^{(r)}\right\| \\
& +\frac{(n+r-1) !(n-r-1) !}{((n-2) !)^{2}} \\
& \times\left(1+\sqrt{\frac{2\left(n+1+2 r^{2}+4 r\right) x^{2}+2\left(n+2 r^{2}+3 r\right) x+r(r+1)}{n-r-2}}\right) \omega\left(f^{(r)},(n-r-1)^{-1 / 2}\right),
\end{aligned}
$$

where $x \in[0, \infty)$. 
Proof. Since $\int_{0}^{\infty} b_{n-r, \nu+r-1}(t) d t=1$ and $\sum_{\nu=0}^{\infty} p_{n, \nu}(x)=1$, we can use Lemma 2.2 to obtain

$$
\begin{aligned}
& G_{n}^{(r)}(f, x)-f^{(r)}(x)=\frac{(n+r-1) !(n-r-1) !}{((n-1) !)^{2}} \sum_{\nu=0}^{\infty} p_{n+r, \nu}(x) \\
\times & \int_{0}^{\infty} b_{n-r, \nu+r-1}(t)\left[f^{(r)}(t)-f^{(r)}(x)\right] d t+\left[\frac{(n+r-1) !(n-r-1) !}{((n-1) !)^{2}}-1\right] f^{(r)}(x) .
\end{aligned}
$$

Taking into account the well known property $\omega\left(f^{(r)}, \lambda \delta\right) \leq(1+\lambda) \omega\left(f^{(r)}, \delta\right)$, $\lambda \geq 0$, we get

$$
\begin{aligned}
& \left|G_{n}^{(r)}(f, x)-f^{(r)}(x)\right| \\
& \leq \frac{(n+r-1) !(n-r-1) !}{((n-1) !)^{2}} \sum_{\nu=0}^{\infty} p_{n+r, \nu}(x) \int_{0}^{\infty} b_{n-r, \nu+r-1}(t)\left|f^{(r)}(t)-f^{(r)}(x)\right| d t \\
& \quad+\left[\frac{(n+r-1) !(n-r-1) !}{((n-1) !)^{2}}-1\right]\left\|f^{(r)}\right\| \\
& \leq \frac{(n+r-1) !(n-r-1) !}{((n-1) !)^{2}} \sum_{\nu=0}^{\infty} p_{n+r, \nu}(x) \\
& \quad \times \int_{0}^{\infty} b_{n-r, \nu+r-1}(t)\left(1+\delta^{-1}|t-x|\right) \omega\left(f^{(r)}, \delta\right) d t \\
& +\left[\frac{(n+r-1) !(n-r-1) !}{((n-1) !)^{2}}-1\right]\left\|f^{(r)}\right\| .
\end{aligned}
$$

Further, using Cauchy's inequality, we have

$$
\begin{aligned}
& \sum_{\nu=0}^{\infty} p_{n+r, \nu}(x) \int_{0}^{\infty} b_{n-r, \nu+r-1}(t)|t-x| d t \\
& \quad \leq\left\{\sum_{\nu=0}^{\infty} p_{n+r, \nu}(x) \int_{0}^{\infty} b_{n-r, \nu+r-1}(r)(t-x)^{2} d t\right\}^{1 / 2}
\end{aligned}
$$

By direct calculations

$$
\int_{0}^{\infty} b_{n-r, \nu+r-1}(t)(t-x)^{2} d t=\frac{(\nu+r)(\nu+r+1)}{(n-r-2)(n-r-1)}-2 x \frac{\nu+r}{n-r-1}+x^{2} .
$$

Hence we have

$$
\sum_{\nu=0}^{\infty} p_{n+r, \nu}(x) \int_{0}^{\infty} b_{n-r, \nu+r-1}(t)(t-x)^{2} d t=\frac{2\left(n+1+2 r^{2}+4 r\right)}{(n-r-2)(n-r-1)} x^{2}
$$




$$
+\frac{2[n+r(2 r+3)]}{(n-r-2)(n-r-1)} x+\frac{r(r+1)}{(n-r-2)(n-r-1)} .
$$

Thus by (12) and (13), we obtain

$$
\begin{aligned}
&\left|G_{n}^{(r)}(f, x)-f^{(r)}(x)\right| \leq \frac{(n+r-1) !(n-r-1) !}{((n-1) !)^{2}} \\
& \times {\left[1+\delta^{-1}\left(\frac{2\left(n+1+2 r^{2}+4 r\right)}{(n-r-2)(n-r-1)} x^{2}\right.\right.} \\
&\left.\left.+\frac{\left.2\left(n+2 r^{2}+3 r\right)\right]}{(n-r-2)(n-r-1)} x+\frac{r(r+1)}{(n-r-2)(n-r-1)}\right)^{1 / 2}\right] \omega\left(f^{(r)}, \delta\right) \\
&+\left[\frac{(n+r-1) !(n-r-1) !}{((n-1) !)^{2}}-1\right]\left\|f^{(r)}\right\| .
\end{aligned}
$$

Choosing $\delta^{-1}=\sqrt{n-r-1}$, we obtain the desired result.

\section{Linear Combinations}

It turns out that the order of approximation by operators (1) is at best $O\left(n^{-1}\right)$, even for smooth functions. With the aim of improving the order of approximation, we consider linear combinations $G_{n}(f, k, x)$ of the operators $G_{d_{j} n}(f, x)$ as

$$
G_{n}(f, k, x)=\sum_{j=0}^{k} C(j, k) G_{d_{j} n}(f, x),
$$

where $d_{0}, d_{1}, \ldots, d_{k}$ are arbitrary and fixed distinct positive integers and

$$
C(j, k)=\prod_{\substack{i=0,0<j<k \\ i \neq j}} \frac{d_{j}}{d_{j}-d_{i}}, \quad k \neq 0, \quad C(0,0)=1 .
$$

Such linear combinations were considered by C. P. May [7] to improve the order of approximation of exponential type operators.

The $m$-th order modulus of continuity $\omega_{m}(f, \delta, a, b)$ for a continuous function $f$ on the interval $[a, b]$ is defined by

$$
\omega_{m}(f, \delta, a, b)=\sup \left\{\left|\Delta_{h}^{m} f(x)\right|:|h| \leq \delta ; \quad x, x+h \in[a, b]\right\} .
$$

For $m=1, \omega_{m}(f, \delta)$ is written simply as an the ordinary modulus of continuity $\omega_{f}(\delta)$ or $\omega(f, \delta)$, see, e.g., [2].

$C_{\gamma}[0, \infty) \equiv\left\{f \in C[0, \infty):|f(t)| \leq M(1+t)^{\gamma}\right.$ for some $\left.M>0\right\}$, where $\gamma>0$. We define the norm $\|f\|_{\gamma}$ on the space $C_{\gamma}[0, \infty)$ by $\|f\|_{\gamma}=\sup _{0 \leq t<\infty}|f(t)|(1+t)^{-\gamma}$. Let us assume that $0<a<a_{1}<b_{1}<b<\infty$ for sufficiently small $\delta>0$, the $(2 k+2)$-th order Steklov mean $f_{2 k+2, \delta}(t)$ corresponding to $f \in C_{\gamma}[0, \infty)$ is 
defined by

$$
f_{2 k+2, \delta}(t)=\delta^{-(2 k+2) m} \int_{-\delta / 2}^{\delta / 2} \int_{-\delta / 2}^{\delta / 2} \cdots \int_{-\delta / 2}^{\delta / 2}\left[f(t)-\Delta_{\eta}^{2 k+2} f(t)\right] \prod_{i=1}^{2 k+2} d t_{i},
$$

where $\eta=\frac{1}{2 k+2} \sum_{i=1}^{2 k+2} t_{i}$ and $t \in[a, b]$.

It is easily checked (see, e.g., [3], [6, Theorem 18.17]) that

(i) $f_{2 k+2, \delta}$ has continuous derivatives up to order $(2 k+2)$ on $[a, b]$;

(ii) $\quad\left\|f_{2 k+2, \delta}^{(r)}\right\|_{C\left[a_{1}, b_{1}\right]} \leq c \delta^{-r} \omega_{r}\left(f, \delta, a_{1}, b_{1}\right), r=1,2, \ldots, 2 k+2$;

(iii) $\left\|f-f_{2 k+2, \delta}\right\|_{C\left[a_{2}, b_{2}\right]} \leq c \omega_{2 k+2}(f, \delta, a, b)$;

(iv) $\quad\left\|f_{2 k+2, \delta}\right\|_{C\left[a_{2}, b_{2}\right]} \leq c\|f\|_{\gamma}$,

where the constants $c$ are independent of $f$ and $\delta$.

Theorem 4.1 (Asymptotic formula). Let $f \in C_{\gamma}[0, \infty)$. If $f^{(2 k+r+2)}$ exists at a point $x \in(0, \infty)$, then

$$
\lim _{n \rightarrow \infty} n^{k+1}\left\{G_{n}^{(r)}(f, k, x)-f^{(r)}(x)\right\}=\sum_{i=r+1}^{2 k+r+2} Q(i, k, r, x) f^{(i)}(x),
$$

where $Q(i, k, r, x)$ are certain polynomials in $x$.

Proof. By using the definition of linear combinations (14), Lemma 2.2 and Taylor's expansion of $f$, we have

$$
\begin{aligned}
n^{k+1} & \sum_{j=0}^{k} C(j, k) \frac{\left(\left(d_{j} n-1\right) !\right)^{2}}{\left(d_{j} n+r-1\right) !\left(d_{j} n-r-1\right) !} G_{d_{j} n}^{(r)}(f, x)-f^{(r)}(x) \\
= & n^{k+1} \sum_{j=0}^{k} C(j, k) \frac{\left(\left(d_{j} n-1\right) !\right)^{2}}{\left(d_{j} n+r-1\right) !\left(d_{j} n-r-1\right) !} \\
& \times G_{d_{j} n}^{(r)}\left(\sum_{i=0}^{2 k+r+2} \frac{f^{(i)}(x)}{i !}(t-x)^{i}+\varepsilon(t, x)(t-x)^{2 k+r+2}, x\right)-f^{(r)}(x) \\
= & n^{k+1} \sum_{i=0}^{2 k+r+2} \frac{f^{(i)}(x)}{i !} \sum_{j=0}^{k} C(j, k) \sum_{\nu=0}^{\infty} p_{d_{j} n+r, \nu}(x) \\
& \times \int_{0}^{\infty} b_{d_{j} n-r, \nu+r-1}(t) \frac{d^{r}}{d x^{r}}(t-x)^{i} d t-f^{(r)}(x) \\
& +n^{k+1} \sum_{j=0}^{k} C(j, k) \sum_{\nu=1}^{\infty} p_{d_{j} n, \nu}^{(r)}(x) \int_{0}^{\infty} b_{d_{j} n, \nu}(t) \varepsilon(t, x)(t-x)^{2 k+r+2} d t \\
= & \sum_{i=r+1}^{2 k+r+2} Q(i, k, r, x) f^{(i)}(x)+E_{n, k, r}(x)+o(1)
\end{aligned}
$$


by Lemma 2.1, where

$$
E_{n, k, r}(x)=n^{k+1} \sum_{j=0}^{k} C(j, k) \sum_{\nu=1}^{\infty} p_{d_{j} n, \nu}^{(r)}(x) \int_{0}^{\infty} b_{d_{j} n, \nu}(t) \varepsilon(t, x)(t-x)^{2 k+r+2} d t
$$

which tends to zero as $n \rightarrow \infty$ (for this proof we refer the reader to [5, Theorem 5]).

Theorem 4.2 (Error Estimation). Let $f^{(r)} \in C_{\gamma}[0, \infty)$ and $0<a<a_{1}<$ $b_{1}<b<\infty$. Then for $n$ sufficiently large, we have

$$
\begin{aligned}
& \left\|G_{n}^{(r)}(f, k, \cdot)-f^{(r)}\right\|_{C\left[a_{1}, b_{1}\right]} \\
& \quad=\max \left\{C(k, r) \omega_{2 k+2}\left(f^{(r)}, n^{-1 / 2}, a, b\right), C(k, r, f) n^{-(k+1)}\|f\|_{\gamma}\right\},
\end{aligned}
$$

where $C(k, r)$ and $C(k, r, f)$ are constants depending on the parameters in parentheses.

Proof. First by the linearity property we have

$$
\begin{aligned}
& \left\|G_{n}^{(r)}(f, k, \cdot)-f^{(r)}\right\|_{C\left[a_{1}, b_{1}\right]} \leq\left\|G_{n}^{(r)}\left(\left(f-f_{2 k+2, \delta}\right), k, \cdot\right)\right\|_{C\left[a_{1}, b_{1}\right]} \\
& +\left\|G_{n}^{(r)}\left(f_{2 k+2, \delta}, k, \cdot\right)-f_{2 k+2, \delta}^{(r)}\right\|_{C\left[a_{1}, b_{1}\right]}+\left\|f^{(r)}-f_{2 k+2, \delta}^{(r)}\right\|_{C\left[a_{1}, b_{1}\right]} \\
& =E_{1}+E_{2}+E_{3}, \quad \text { say. }
\end{aligned}
$$

By property (iii) of the Steklov mean we have

$$
E_{3} \leq c \omega_{2 k+2}\left(f^{(r)}, \delta, a, b\right)
$$

Next using Theorem 4.1, we have

$$
E_{2} \leq c n^{-(k+1)} \sum_{j=r}^{2 k+r+2}\left\|f_{2 k+2, \delta}^{(j)}\right\|_{C[a, b]}
$$

By applying the interpolation property due to Goldberg and Meir [4] for each $j=r, r+1, \ldots, 2 k+r+2$, we have

$$
\left\|f_{2 k+2, \delta}^{(j)}\right\|_{C[a, b]} \leq c\left\{\left\|f_{2 k+2, \delta}\right\|_{C[a, b]}+\left\|f_{2 k+2, \delta}^{(2 k+r+2)}\right\|_{C[a, b]}\right\} .
$$

Therefore, applying properties (ii) and (iv) of Steklov mean, we obtain

$$
E_{2} \leq c n^{-(k+1)}\left\{\|f\|_{\gamma}+\delta^{-(2 k+2)} \omega_{2 k+2}\left(f^{(r)}, \delta\right)\right\} .
$$

Finally, we estimate $E_{1}$, choosing $a^{*}, b^{*}$ satisfying the condition $0<a<a^{*}<$ $a_{1}<b_{1}<b^{*}<b<\infty$. Also let $\psi(t)$ denote the characteristic function of the interval $\left[a^{*}, b^{*}\right]$, then

$$
\begin{aligned}
E_{1} \leq \| & G_{n}^{(r)}\left(\psi(t)\left(f(t)-f_{2 k+2, \delta}(t)\right), k, \cdot\right) \|_{C\left[a_{1}, b_{1}\right]} \\
& +\left\|G_{n}^{(r)}\left((1-\psi(t))\left(f(t)-f_{2 k+2, \delta}(t)\right), k, \cdot\right)\right\|_{C\left[a_{1}, b_{1}\right]}=E_{4}+E_{5}, \quad \text { say. }
\end{aligned}
$$

We may note here that to estimate $E_{4}$ and $E_{5}$, it is enough to consider their expressions without linear combinations. By Lemma 2.2 we have 


$$
\begin{aligned}
& G_{n}^{(r)}\left(\psi(t)\left(f(t)-f_{2 k+2, \delta}(t)\right), x\right) \\
= & \frac{(n+r-1) !(n-r-1) !}{((n-1) !)^{2}} \sum_{\nu=0}^{\infty} p_{n+r, \nu}(x) \int_{0}^{\infty} b_{n-r, \nu+r-1}(t)\left|f^{(r)}(t)-f_{2 k+2, \delta}^{(r)}(t)\right| d t .
\end{aligned}
$$

Hence

$$
\left\|G_{n}^{(r)}\left(\psi(t)\left(f(t)-f_{2 k+2, \delta}(t)\right), k, \cdot\right)\right\|_{C\left[1_{1}, b_{1}\right]} \leq c\left\|f^{(r)}-f_{2 k+2, \delta}\right\|_{C\left[a^{*}, b^{*}\right]} .
$$

Now for $x \in\left[a_{1}, b_{1}\right]$ and $t \in[0, \infty) \backslash\left[a^{*}, b^{*}\right]$, we choose $\delta_{1}>0$ satisfying $|t-x| \geq$ $\delta_{1}$. Therefore by Lemma 2.4 and Schwarz inequality, we have

$$
\begin{aligned}
& I=\left|G_{n}^{(r)}\left((1-\psi(t))\left(f(t)-f_{2 k+2, \delta}(t)\right), x\right)\right| \\
& \leq \sum_{\substack{2 i+j \leq r \\
i, j \geq 0}} n^{i} \frac{\left|\phi_{i, j, r}(x)\right|}{\{x(1+x)\}^{r}} \sum_{\nu=1}^{\infty} p_{n, \nu}(x)|\nu-n x|^{j} \\
& \times \int_{0}^{\infty} b_{n, \nu-1}(t)(1-\psi(t))\left|f(t)-f_{2 k+2, \delta}(t)\right| d t \\
& +(1+x)^{-n-r} n(n+1) \cdots(n+r-1)(1-\psi(0))\left|f(0)-f_{2 k+2, \delta}(0)\right| \\
& \leq c\|f\|_{\gamma}\left\{\sum_{\substack{2 i+j \leq r \\
i, j \geq 0}} n^{i} \sum_{\nu=1}^{\infty} p_{n, \nu}(x)|\nu-n x|^{j} \int_{|t-x| \geq \delta_{1}} b_{n, \nu-1}(t) d t\right. \\
& \left.+(1+x)^{-n-r} n(n+1) \cdots(n+r-1)\right\} \\
& \leq c\|f\|_{\gamma}\left\{\delta_{1}^{2 s} \sum_{\substack{2 i+j \leq r \\
i, j \geq 0}} n^{i} \sum_{\nu=1}^{\infty} p_{n, \nu}(x)|\nu-n x|^{j}\left(\int_{0}^{\infty} b_{n, \nu-1}(t) d t\right)^{1 / 2}\right. \\
& \left.+\left(\int_{0}^{\infty} b_{n, \nu-1}(t)(t-x)^{4 s} d t\right)^{1 / 2}+(1+x)^{-n-r} n(n+1) \cdots(n+r-1)\right\} \\
& \leq c\|f\|_{\gamma} \delta_{1}^{-2 s} \sum_{\substack{2 i+j \leq r \\
i, j \geq 0}} n^{i}\left\{\sum_{\nu=0}^{\infty} p_{n, \nu}(x)(\nu-n x)^{2 j}-(1+x)^{-n}(-n x)^{2 j}\right\}^{1 / 2} \\
& \times\left\{\sum_{\nu=0}^{\infty} p_{n, \nu}(x) \int_{0}^{\infty} b_{n, \nu-1}(t)(t-x)^{4 s} d t-(1+x)^{-n}(-x)^{4 s}\right\}^{1 / 2} \\
& +c\|f\|_{\gamma}(1+x)^{-n-r} n(n+1) \cdots(n+r-1) .
\end{aligned}
$$

Hence, by Lemma 2.1 and Lemma 2.3, we have

$$
I \leq c\|f\|_{\gamma} \delta_{1}^{-2 m} O\left(n^{\left(i+\frac{j}{2}-s\right)}\right) \leq c n^{-q}\|f\|_{\gamma}, \quad q=s-r / 2
$$


where the last term vanishes as $n \rightarrow \infty$. Now choosing $m>0$ satisfying $q \geq k+1$, we obtain

$$
I \leq c n^{-(k+1)}\|f\|_{\gamma} .
$$

Therefore by property (iii) of the Steklov mean, we get

$$
\begin{aligned}
E_{1} & \leq c\left[\left\|f^{(r)}-f_{2 k+2, \delta}^{(r)}\right\|_{C\left[a^{*}, b^{*}\right]}+n^{-(k+1)}\|f\|_{\gamma}\right] \\
& \leq c\left[\omega_{2 k+2}\left(f^{(r)}, \delta, a, b\right)+n^{-(k+1)}\|f\|_{\gamma}\right] .
\end{aligned}
$$

Setting $\delta=n^{-1 / 2}$, the theorem follows.

\section{ACKNOWLEDGEMENT}

The author is thankful to the referee for making valuable suggestions leading to a better presentation of the paper.

\section{REFERENCES}

1. R. A. DeVore and G. G. Lorentz, Constructive approximation. Grundlehren der Mathematischen Wissenschaften [Fundamental Principles of Mathematical Sciences], 303. Springer-Verlag, Berlin, 1993.

2. Z. Ditzian and V. Tотік, Moduli of smoothness. Springer Series in Computational Mathematics, 9. Springer-Verlag, New York, 1987.

3. G. Freud and V. Popov, On approximation by Spline functions. Proceeding Conference on Constructive Theory Functions, 163-172, Budapest, 1969.

4. S. Goldberg and V. Meir, Minimum moduli of ordinary differential operators. Proc. London Math. Soc. (3) 23(1971), 1-15.

5. V. Gupta, M. K. Gupta and V. Vasishtha, Simultaneous approximation by summation-integral type operators. Nonlinear Funct. Anal. Appl. 8(2003), No. 3, 399412.

6. E. Hewitt and K. Stromberg, Real and abstract analysis. A modern treatment of the theory of functions of a real variable. Springer-Verlag, New York, 1965.

7. C. P. MAY, Saturation and inverse theorems for combinations of a class of exponentialtype operators. Canad. J. Math. 28(1976), No. 6, 1224-1250.

8. R. P. Sinha, P. N. Agrawal and V. Gupta, On simultaneous approximation by modified Baskakov operators. Bull. Soc. Math. Belg. Sér. B 43(1991), No. 2, 217-231.

9. H. M. SRIVAstava and V. Gupta, A certain family of summation-integral type operators. Math. Comput. Modelling 37(2003), No. 12-13, 1307-1315.

(Received 19.03.2004; revised 15.09.2004)

Author's address:

School of Applied Sciences

Netaji Subhas Institute of Technology

Sector 3 Dwarka, New Delhi 110045

India

E-mail: vijay@nsit.ac.in 